\title{
Molecular studies into Copine-4 function in Retinal Ganglion Cells
}

$5 \quad{ }^{1}$ Retinal Circuit Development \& Genetics Unit, Neurobiology Neurodegeneration \& Repair

6 Laboratory, NEI, National Institutes of Health, Bethesda, Maryland 20892

$7 \quad 2$ Department of Neuroscience, College of Medicine, The Ohio State University, 333 W 10th

8 Avenue, Columbus, Ohio 43210

$9 \quad{ }^{3}$ Proteomics Core, NHLBI, National Institutes of Health, Bethesda, Maryland 20892

$10{ }^{4}$ Section on Molecular Structure and Functional Genomics, NEI, National Institutes of Health,

11 Bethesda, Maryland 20892

$12{ }^{5}$ Research and Development Institute, Faculty of Medicine, Transilvania University of Brasov,

13 Brasov, Romania

$15 *$ Corresponding author: Tudor C. Badea,

16 Retinal Circuit Development \& Genetics Unit, Building 6, Room 331, 6 Center Drive, Bethesda,

17 MD 20892-0610. / Research and Development Institute, Transilvania University of Brasov,

18 Blvd. Eroilor Nr. 29, Brasov, cod 500036, Romania.

19 Email: badeatc@mail.nih.gov / badeatc@unitbv.ro 


\section{ABSTRACT}

The molecular mechanisms underlying morphological diversity in retinal cell types are

27 poorly understood. We have previously reported that several members of the Copine family of

28 Ca-dependent membrane adaptors are expressed in Retinal Ganglion Cells (RGCs) and

29 transcriptionally regulated by Brn3 transcription factors. Several Copines are enriched in the

30 retina and their over-expression leads to morphological changes reminiscent of neurite formation

31 in HEK293 cells. However, the role of Copines in the retina is largely unknown. Here we focus

32 on Cpne4, a Copine whose expression is restricted to RGCs. Over-expression of Cpne4 in RGCs

33 in vivo led to formation of large varicosities on the dendrites but did not otherwise visibly affect

34 dendrite or axon formation. Protein interactions studies using yeast two hybrid analysis from

35 whole retina cDNA revealed two Cpne4 interacting proteins - HCFC1 and Morn2. Mass

36 Spectrometry analysis of retina lysate pulled down using Cpne4 or its vonWillebrand A (vWA)

37 domain identified a further 207 interacting proteins. Gene Ontology (GO) analysis of Cpne4

38 interactors suggests its involvement in several metabolic and signaling pathways, including

39 processes related to vesicle trafficking, intracellular membrane bound organelles, and plasma

40 membrane associated structures, including neurites. We conclude that, consistent with its domain

41 structure, Cpne4 may be involved in assembly and trafficking of several membrane associated

42 cell compartments. 


\section{INTRODUCTION}

RGCs in the retina transmit visual signals received by photoreceptors to the brain for

processing visual inputs. Different RGC sub-types are responsible for computing different

51 aspects of the visual stimuli. The combinatorial expression of transcription factors in different

52 RGC sub-types regulates cell specific morphologies and physiology by controlling molecules

53 involved in the development of dendrite/axon morphology, synapse formation and function $(1,2)$.

How cell-specific morphologies develop in the retina is not well understood. One likely

55 mechanism is that transcription factors encode specific morphological features via adhesion

56 molecules or cytoskeletal elements they regulate. For example, Tbr1 regulates cell adhesion

57 molecules Cdh8 and Sorcs3 resulting in dendritic stratification of J-RGCs in the Off sublamina

58 (3). Other such molecules might be responsible for cell specific morphologies in other RGC

59 types. Copines are a family of such candidate cell morphology determinants, regulated by Brn3

60 transcription factors. Copines consist of two C2 domains (C2A and C2B) and a vWA domain

61 (4,5). The Copine C2 domains are similar to those found in a variety of vesicular traffic proteins

62 such as Synaptotagmins, Munc18, Rabphilin3A and Doc2, and have been involved in calcium

63 dependent binding to cell membranes (6). vWA domains are typically found extracellularly in

64 several proteins (e.g. integrins) and are involved in protein-protein interactions (7). However

65 Copines (and their vWA domain) are intracellular proteins that interact transiently with the inner

66 leaflet of the plasma membrane.

67 There are nine Copines in mammals- Cpne1- 9 (4,8-10). Of these, Cpne1, 2 and 3 are

68 expressed ubiquitously. Cpne4, 5, 6, 7, 8 and 9 are enriched in neurons. Cpne5 and 8 are also

69 expressed in other tissues such as kidneys, lungs, testes, mammary glands $(11,12)$. 
Copines are conserved across several species. They have previously been shown to be

71 important in a variety of cellular functions. Copines are involved in myofilament stability in C.

72 elegans and plant growth in Arabidopsis $(13,14)$. Copines are also involved in cytokinesis and

73 contractile vacuole function by regulating cAMP signaling in Dictyostelium (15). Copine has

74 also been shown to interact with actin filaments to regulate chemotaxis and adhesion in

75 Dictyostelium (16).

In the nervous system, Copines were first seen to be localized in hippocampus and

77 olfactory bulb neurons in mouse brain $(10,17)$. Cpne6 is expressed in the hippocampus and is

78 required for regulating the spine morphology during long term potentiation in hippocampus by

79 regulating the Rac-LIMK-Cofilin and BDNF-TrkB pathways $(18,19)$. Cpne1 has been previously

80 seen to be upregulated during development and is required for hippocampal progenitor cell

81 differentiation into neurons $(20,21)$. Cpne7 is expressed in sublaterodorsal nucleus in pontine

82 segmental area and is required for REM sleep (22).

83 In the retina, we have previously reported that Cpne4, 5, 6 and 9 are enriched in the inner

84 retina and they are regulated by Brn3b and $\operatorname{Brn} 3 a(1,23)$. Whereas Cpne5, 6 and 9 were

85 expressed in most of the GCL as well as INL, Cpne4 is the only Copine specifically expressed in

86 RGCs (with the exception of one INL amacrine cell type) (23). Using over-expression studies in

87 HEK293 cells, we found that Copines can significantly alter cell morphology, inducing

88 elongated membrane processes similar to neurites. In the current study, we further explore the

89 effects of Cpne4 expression in RGCs and study its protein interactions using yeast two hybrid

90 analysis and Mass Spectrometry, to identify its cell biological functions and role in RGCs.

\section{2. MATERIALS AND METHODS}




\subsection{Transfection in HEK293-Cre cells}

The cDNA for full length Cpne4, two C2 domain or vWA domain were cloned into pAAV-FLEX-HA-T2A-meGFP plasmid vector (Fig.1A). The cDNA is in frame with 3XHA (3

96 tandem copies of Hemagglutinin antigen tag- 5' TACCCATACGATGTTCCAGATTACGCT 3'

97 or 5' TATCCATATGATGTTCCAGATTATGCT 3') and separated from meGFP (membrane

98 eGFP) by a P2A peptide sequence. The constructs were transfected into HEK293-Cre cells (a

99 kind gift of Dr. Brian Sauer) [1, 23, 62] using Lipofectamine (Invitrogen, Carlsbad, CA). The

100 transfected cells were fixed in $2 \% \mathrm{PFA}$ after 48 hours and processed for immunofluoroscence as

101 described in section 2.4.

102

\subsection{Mouse lines}

Adult $B r n 3 b^{K O / K O}$ (or KO) (24) and $B r n 3 b^{W / W T}$ (or WT) littermates were used for

105 immunohistochemistry (IHC). Postnatal day 0 (P0) or P14 Brn3b $b^{C r e / W T}$ mice were used for AAV1

106 virus infections. Adult wild-type (C57/B16 - SV129 mixed background) mice were used for

107 retina pulldowns and mass spectrometry experiments, to determine the protein interactors of

108 Cpne4. All animal procedures were approved by the National Eye Institute (NEI) Animal Care 109 and Use Committee under protocol NEI640.

\section{$112 \quad 2.3$ Virus infections in retina}

113 Flex-meGFP-P2A-HA-Cpne4, described in section 2.1, was packaged into AAV1 viruses

114 (henceforth AAV1-Cpne4). Intraocular injections of 0.5 ul (1e9 viral particles/ul) of AAV1115 Cpne4 or AAV1-eGFP control virus were done in P0 or P14 Brn3bCre/WT mice eyes using 
116 glass capillaries fitted onto a Femtojet device (Eppendorf, Enfield, CT), as previously described.

117 Injections were aimed at the scleral region adjacent to the limbus. Eyes were collected at 2-3

118 months of age and flat-mounted for immunostaining. The eyes were fixed in $4 \%$

119 paraformaldehyde for 15 minutes and retinas were dissected to make a flat mount preparation.

120 The retinas were again fixed for 30 minutes and then washed three times with PBS+ $0.5 \%$

121 Triton-X 100 (PBST). Immunofluorescence was performed as described below.

\subsection{Immunofluorescence}

Brn3b WT and KO sections were co-immunostained to confirm the presence of different

125 interactor proteins identified from yeast two hybrid and mass spectrometry analysis in the retinal

126 ganglion cells. Similar process was followed for immunofluorescence of transfected HEK293

127 cells on coverslips. The sections or cells were incubated with blocking solution- $10 \%$ bovine

128 serum albumin (BSA), 10\% normal donkey serum (NDS) and 0.5\% Triton X 100, for one hour at

129 room temperature. The blocking solution was then replaced with primary antibody solution

130 containing the antibodies at the required concentrations and incubated overnight, at $4^{0} \mathrm{C}$. Sections

131 or cells were washed three times with PBST and incubated with the secondary antibody solutions

132 for one hour at room temperature. The sections or cells were washed again with PBST and 133 coverslipped.

134 For staining the AAV1 infected retina flatmounts, the retinas were incubated in blocking 135 solution, overnight at $4^{0} \mathrm{C}$. The blocking solution was then replaced with primary antibody 136 solution and retinas incubated for 48 hours at $4^{0} \mathrm{C}$. The retinas were then washed three times in

137 PBST, secondary antibody solution was added and retinas were incubated overnight at $4^{0} \mathrm{C}$. The 138 retinas were washed again three times with PBST, carefully mounted on glass slides and 
139 coverslips were placed. Image acquisition was on either a Axioimager Z2 fitted with an apotome

140 device, or on a LSM 880 confocal microscope (both from Zeiss, White Plains, NY). The images

141 were taken as 1um thick z-stacks and the images were stacked using ImageJ. Colocalization

142 analysis of Copine4-interacting proteins in HEK293 cells was performed using the "Coloc 2"

143 plugin in Image J.

\subsection{Yeast two hybrid}

A Gal4 based yeast two hybrid analysis was performed to identify the proteins that interact with Cpne4 vWA domain. An adult mouse retina cDNA library was cloned in pGADT7

148 (carrying a Trp1 selection gene) and transformed in AH109 yeast strain (containing His3, Ade2,

149 lacZ and Mel1 selections; Clontech, BD Biosciences, Pao Alto, CA). Cpne4 vWA domain was 150 cloned into pGBKT7 (carrying Leu2 selection gene) and transformed in to competent Y187 yeast

151 strain (lacZ and Mel1 selection). The yeast mating experiment was performed as per the Two-

152 hybrid library screening protocol for yeast mating (Clontech). Briefly, one colony of Y187 153 transformed with pGBKT7+ Cpne4 vWAdomain was inoculated in $50 \mathrm{ml}$ of Tryptophan (Trp)

154 selection media and incubated at $30^{\circ} \mathrm{C}$ overnight. The following day, the culture media was 155 centrifuged, and the pellet re-suspended in $5 \mathrm{ml}$ Trp selection media. $45 \mathrm{ml}$ of YPDA media (YPD 156 media supplemented with adenine(Ade)) was added to it. $1 \mathrm{ml}$ of cDNA library (titer $=5 \mathrm{x}$ $15710^{7} \mathrm{cfu} / \mathrm{ml}$ ) was thawed in a water bath at room temperature and added to the above and allowed 158 to mate for approximately 24 hours at $30^{\circ} \mathrm{C}$ with slow shaking at 50rpm. The next day, the 159 presence of mated, diploid yeast cells was checked under light microscope. The culture was then 160 centrifuged, and the pellet resuspended in $15 \mathrm{ml} 0.5 \mathrm{X}$ YPDA. 300ul aliquots of the entire $15 \mathrm{ml}$ 161 mating culture was spread on $15 \mathrm{~cm}$ quadruple selection agar plates (with selection for Trp, 
162 leucine (Leu), Ade and histidine (His) and X-alpha-Gal reporter) and grown for about 5 days at $16330^{\circ} \mathrm{C}$. Small scale positive and negative control matings were also performed. For the positive

164 control, pGBKT7-53 encoding the p53 protein and pGADT7-T encoding the SV40 large T 165 antigen protein were transformed into Y187 and AH109, respectively. For negative control, 166 empty pGBKT7 and pGADT7, with no gene insertions, were transformed into Y187 and AH109, 167 respectively. For the positive control mating, one colony each from Y187 + pGBKT7-53 and 168 AH109 + pGADT7-T were inoculated in 500ul 2X YPDA and incubated overnight at 30 degrees 169 at 200rpm. Similarly, for the negative control mating, Y187 + empty pGBKT7 and AH109 + 170 pGADT7 were inoculated in 500ul 2X YPDA and incubated overnight at 30 degrees at 200rpm.

171 The following day the control cultures were spread in 1:10, 1:100 and 1:1000 dilutions on 172 separate single (Leu or Trp), double (Leu and Trp) or quadruple selection agar plates and grown 173 for $3-5$ days at $30^{\circ} \mathrm{C}$.

174 After 4 days of selection, 241 pale blue colonies were picked for confirmation. They were 175 streaked separately on fresh agar plates with quadruple selection. Six colonies grew into blue 176 colonies after 3-4 days. For both positive and negative controls, the colonies appeared on the 177 single selection and double selection plates. But on the quadruple selection plates, blue colonies 178 appeared only on the positive control and there were no colonies for the negative control.

179 Colony PCR was performed on the six selected colonies, and PCR products were extracted from 180 the gel and Sanger sequenced (Eurofins, Luxembourg). Inserts were identified by BLAST 181 searches against the NCBI mouse transcriptome collection. 
HEK293-Cre cells were co-transfected with HA-eGFP-Cpne4-vWAdomain construct

185 (described in section 2.1) and Flag- tagged target protein or protein domain identified from yeast

186 two hybrid analysis. 24 hours after the transfection, the cells were washed three times with $1 \mathrm{X}$

187 PBS. 300ul lysis buffer (50mM Tris-HCl, 150mM NaCl, 0.5\% NP40 and 1mM EDTA)

188 containing protease inhibitor (Roche, Basel, Switzerland) and 0.2M PMSF was added to each

189 well and incubated on ice for 15 minutes. The lysate was then collected in $1.5 \mathrm{ml}$ tubes and

190 centrifuged at $14000 \mathrm{~g}$ for 10 minutes to remove any debris. Meanwhile, magnetic beads were

191 washed with 1X PBS and incubated with Flag antibody for 30 minutes, with end-to-end rotation

192 at $4^{0} \mathrm{C}$. The beads were again washed with 1 X PBS to remove any unbound antibody and the

193 supernatant from the cell lysate was added to it. These were incubated overnight with end-to-end

194 rotation at $4^{0} \mathrm{C}$. The next day, the beads were washed four times and PBS + Laemmli buffer

195 (62.5mM Tris-HCl, 2\% SDS, 10\% glycerol, 5\% beta-mercapto-ethanol, bromophenol blue) was

196 added to the beads. The beads were boiled for 5 minutes at $70^{\circ} \mathrm{C}$. The beads were separated on a

197 magnetic rack and the supernatant loaded on a 10\% SDS-PAGE gel. The gel was allowed to run

198 until the dye front reached the bottom of the gel. The separated proteins were then transferred to

199 0.2um PVDF membranes and processed further for Western blotting.

\subsection{GST pulldown from retina}

GST tagged Cpne4 protein was synthesized from bacteria as described before (23). GST-

203 Cpne4-vWAdomain and GST proteins were also synthesized similarly. 19 wild-type retinas

204 (C57B16 and SV129) were homogenized using a glass homogenizer, in cold lysis buffer (RIPA

205 buffer: 50mM Tris-HCl, 150mM NaCl, 1mM EDTA, Complete protease inhibitor (Millipore

206 Sigma, Burlington, MA; Catalog no. 11697498001)). NP40 was then added to the lysate to a 
$2070.5 \%$ final concentration. Glutathione- tagged magnetic beads were added to the lysate and

208 incubated at 4 degrees with end-to-end rotation for 2 hours. The magnetic beads were removed,

209 and the lysate centrifuged at $700 \mathrm{~g}$ for 10 minutes to remove any debris. The lysate was then kept

210 on ice until further process. Equimolar amounts of GST, GST-vWA domain and GST-Cpne4

211 were incubated with glutathione tagged magnetic beads and incubated with end-to-end rotation at

$212 \quad 4^{0} \mathrm{C}$ for 2 hours. The supernatant was discarded, and beads washed three times with PBS. Equal

213 volume of cleared retina lysate (600ul each) was added to each of the above three tubes and

214 incubated overnight at $4^{0} \mathrm{C}$ with end-to-end rotation. The following day, the supernatant was

215 discarded, and the beads were washed four times with PBS. 30ul of glutathione elution buffer

216 was added to each of the tubes and incubated at room temperature for 10 minutes with end-to-

217 end rotation. Laemmli buffer was added and the samples boiled at $70^{\circ} \mathrm{C}$ for 5 minutes. The beads

218 were separated, and the samples loaded onto 4-15\% gradient SDS-PAGE gels. The samples were

219 allowed to run on the gel until the dye front reached the bottom and proteins were visualized

220 using Coomassie blue staining. Full lanes were cut out of the gel for each of the three samples,

221 cut into smaller band size pieces and collected in separate tubes. Three such replicates were

222 prepared each for GST, GST-Cpne4-vWAdomain and GST-Cpne4 pulldowns. The cut bands

223 were then processed further for liquid chromatography mass spectrometry (LC-MS). An

224 additional three replicates also prepared similarly, transferred to PVDF membranes directly after

225 SDS-PAGE and checked with specific antibodies by western blotting. See the section on western

226 blotting for further details. 
For sample preparation for LC-MS, Coomassie blue stained protein gel bands were first 230 destained with $30 \%$ ethanol solution until the gel pieces became transparent. The gel pieces were 231 then destained in $65 \%$ methanol $+10 \%$ acetic acid solution for 30 minutes. The destaining 232 solution was removed and bands were washed with $100 \mathrm{mM}$ TEAB (tri-ethyl ammonium

233 bicarbonate) solution for 5 minutes. 250 ul dehydration solution ( $75 \%$ acetonitrile in $100 \mathrm{mM}$

234 TEAB) was added and the bands were agitated for 10 minutes at room temperature. The 235 dehydration solution was removed, and the bands were air-dryed for a couple of minutes. The gel 236 pieces were then dehydrated with reducing solution (10 mM TCEP in $100 \mathrm{mM}$ TEAB) for 45 237 minutes at $56^{\circ} \mathrm{C}$. This solution was replaced by dehydration solution and bands incubated for 10 238 minutes at room temperature. The bands were then air dried and alkylation solution $(20 \mathrm{mM}$ 239 iodoacetamide in $100 \mathrm{mM}$ TEAB) was added and incubated for 30 minutes at room temperature.

240 The gel bands were washed again and dehydrated again in the dehydration solution until the gel

241 bands shrunk to half the size. The gel bands were air dried briefly and trypsin solution was added 242 to the bands, enough to cover the bands. The bands were left on ice for 5 minutes and more 243 trypsin was added as needed. After 5 minutes, the trypsin solution was removed and $100 \mathrm{mM}$ 244 TEAB was added to tubes, enough to cover the bands. The tubes were incubated at $37^{\circ} \mathrm{C}$ for 245 overnight digestion. The next day, the trypsin solution was removed, and digested peptides 246 transferred to new tubes separately for each sample. 150ul of extraction solution (75\% 247 acetonitrile, $0.1 \%$ formic acid) was then added to each of the tubes and agitated for 10 minutes at 248 room temperature. A short spin was done at 10,000 $\mathrm{g}$ and the gel pieces were saved. The extracts 249 were then vacuum dried and 25 ul $1 \%$ trifluoroacetic acid was added to each tube. This was 250 followed by a peptide clean-up as per the zip-tip protocol (Millipore Sigma; Cat\# C18 251 ZTC18S008). 20ul of $0.1 \%$ formic acid (in acetonitrile) was aspirated in the zip-tip and 
252 discarded. $20 \mathrm{ul}$ of $0.1 \%$ formic acid (in water) was similarly aspirated and discarded. The 253 peptide extract was then aspirated 7-10 times, to let the peptides bind to the zip-tip column. This

254 was followed by washing the zip-tip three times by aspiring $20 \mathrm{ul}$ of wash solution $(0.1 \%$ formic 255 acid in water) and dispensing it. To elute the bound peptides, 20ul of elution buffer (75\% 256 acetonitrile $+0.1 \%$ formic acid) was aspirated and dispensed in a tube. This step was repeated 257 five times and each time eluate was collected in the same tube. The tubes were then dried in a 258 speed vacuum. 20ul of $2 \%$ acetonitrile $+0.1 \%$ formic acid solution was added to the dried 259 peptide digest and the tubes were vortexed and centrifuged. The solution was then transferred to 260 LC vials for LC-MS analysis.

Desalted tryptic peptides were analyzed using nanoscale liquid chromatography tandem 262 mass spectrometry (nLC- MS/MS) and Ultimate 3000-nLC online coupled with an Orbitrap 263 Lumos Tribrid mass spectrometer (Thermo Fisher Scientific, Waltham, MA). Peptides were 264 separated on an EASY-Spray Column (Thermo Fisher Scientific; $75 \mu \mathrm{m}$ by $50 \mathrm{~cm}$ inner 265 diameter, $2-\mu \mathrm{m}$ particle size, and $100-\AA$ pore size). Separation was achieved by 4 to $35 \%$ linear 266 gradient of acetonitrile $+0.1 \%$ formic acid for 90 minutes. An electrospray volt- age of $1.9 \mathrm{kV}$ 267 was applied to the eluent via the EASY-Spray column electrode. The Orbitrap Lumos was 268 operated in positive ion data- dependent mode. Full-scan MS was performed in the Orbitrap with 269 a normal precursor mass range of 380 to $1500 \mathrm{~m} / \mathrm{z}$ (mass/charge ratio) at a resolution of 120,000.

270 The automatic gain control (AGC) target and maximum accumulation time settings were set to 4 $271 \times 10^{5}$ and $50 \mathrm{~ms}$, respectively. MS was triggered by selecting the most intense precursor ions 272 above an intensity threshold of $5 \times 10^{3}$ for collision-induced dissociation (CID)-MS 273 fragmentation with an AGC target and maximum accumulation time settings of $5 \times 10^{3}$ and 300 $274 \mathrm{~ms}$, respectively. Mass filtering was performed by the quadrupole with $1.6 \mathrm{~m} / \mathrm{z}$ transmission 
275 window, followed by CID fragmentation in the ion trap (rapid mode) and collision energy of

$27635 \%$. To improve the spectral acquisition rate, parallelizable time was activated. The number of

277 MS spectra acquired between full scans was restricted to a duty cycle of 3s.

278 Raw data files were processed with the Proteome Discoverer software (v2.4, Thermo

279 Fisher Scientific), using Sequest HT (Thermo Fisher Scientific) search node for carbamylated

280 peptide/protein identifications. The following search parameters were set: protein database

281 UniProtKB/Swiss-Prot Mus musculus (17,033 sequences release 2020_10) concatenated with

282 reversed copies of all sequences; MS1 tolerance of $12 \mathrm{ppm}$ : ion trap detected MS/MS mass

283 tolerance of $0.5 \mathrm{Da}$; enzyme specificity set as trypsin with maximum two missed cleavages;

284 minimum peptide length of 6 amino acids; fixed modification of Cys (carbamidomethylation);

285 variable modification of methionine oxidation and acetyl on $\mathrm{N}$ terminus of protein. Percolator

286 algorithm (v.3.02.1, University of Washington) was used to calculate the false discovery rate

287 (FDR) of peptide spectrum matches (PSM), set to a q-value $<0.05(2-5)$

289 Copine4-vWA domain, we compared the peptides pulled down by either GST-vWA, GST-

290 Copine4 or GST control identified in the previous step. Of the 2119 proteins pulled down in

291 either of the three conditions, we selected for further analysis those that were represented in all

292 three replicates of at least one condition. Differential display analysis was performed using the R

293 package "DEP” (DEP 1.12.0, 10.18129/B9.bioc.DEP; (25)). Missing values were replaced with

294 0, and data was normalized using VSN normalization. Pairwise comparisons for GST only vs.

295 GST-Cpne4 and GST-vWA samples was performed at thresholds of 0.05 FDR and 2-fold

296 change. 


\subsection{Western blotting}

Western blotting (WB) was done as described before (23). Briefly, the PVDF membranes were washed with Tris buffered saline with $0.1 \%$ Tween20 (TBST). The membranes were then incubated in 5\% milk (in TBST) for 1 hour at room temperature. Primary antibody solution

302 prepared in 5\% milk was added to the respective membranes and incubated overnight at $4 \mathrm{C}$ on a 303 rocker shaker. The next day, the membranes were washed three times in TBST and secondary 304 antibody solution (in 5\% milk) was added. The membranes were then incubated at room 305 temperature for 1 hour, followed by three washes with TBST. The membranes were exposed to 306 Super signal chemiluminescence (Thermo Fisher Scientific) for 5 minutes and images taken on a 307 gel dock (Bio-Rad, Hercules, CA).

\subsection{Statistical analysis}

The statistical analysis for comparing the varicosity areas in Cpne4 with control infected

311 RGCs was done using Kolmogorov-Smirnov test (KS2, Matlab). For measuring colocalization of 312 Cpne4 to interacting proteins in co-transfected HEK293 cells, Spearmann's correlation 313 coefficient was used. Number of images, animals and cells measured and p-values are given in 314 the corresponding Results sections.

\section{3. RESULTS}

\section{3.1 Cpne4 transfection induces morphology changes in-vitro}

To evaluate the subcellular distribution of Copine4 or its domains, we transfected 320 expression vectors containing full length Cpne4, C2 domains or vWA domain tagged with HA 
321 (Fig.1A, B), in conjunction with a plasma membrane attached eGFP variant (meGFP) into

322 HEK293 cells. Full length Copine4, C2 domains or vWA domain were detected in the nuclei,

323 cell body as well as plasma membrane of the cells (Fig.1C) as indicated by both HA (red) and

324 Cpne4 (white) staining. As previously reported, full length Cpne4 induces morphological

325 changes (Fig. 1C top panel; $n=2$ experiments) resembling neurites (23). C2 domain or vWA

326 domain constructs also induce extended process formation occasionally, although morphological

327 changes were not as prominent and wide spread as with Cpne4 transfection (Fig.1C middle and

328 bottom panel; $\mathrm{n}=2$ experiments each).

\subsection{Cpne4 infection induces morphology changes in-vivo in RGCs}

Given the morphological defects observed when expressing Copines in HEK293 cells in

332 culture, we asked whether overexpressing Cpne4 in RGCs will affect RGC morphology. We

333 therefore infected either P0 or P14 Brn3b $\mathrm{b}^{\mathrm{Cr} / \mathrm{WT}}$ mice with AAV1 vectors co-expressing HA-

334 tagged Cpne4 and meGFP in a Cre-dependent manner. Membranes from infected Brn3b ${ }^{\text {Cre/WT }}$

335 RGCs were labelled with eGFP (green), revealing axonal arbors, cell bodies and dendrites

336 (Fig.2A, B). In contrast, Cpne4 expression as seen by HA (red) labeling was largely confined to

337 the cell body and dendrites, and only rarely reached into the axon (Fig.2A, B; n=6 retinas, 72

338 cells). Interestingly, several large varicosities were observed on dendrites of Cpne4 infected

339 Brn3b ${ }^{\text {Cre/WT }}$ RGCs. These large varicosities (we call these 'blebs', Fig. 2A, 2A1) can be easily

340 distinguished from regular varicosities, as seen on the RGC dendrites in Cpne4 infected cells

341 (Fig. 2A, 2A2, supplementary Figure 1A, n=6 animals, 72 cells), or on the dendrites of control

342 RGCs infected solely with AAV1-eGFP (Fig.2C, E; area= 10.7 \pm 0.61 um $^{2}$ for Cpne4 versus

$3433.12 \pm 0.18 \mathrm{um}^{2}$ for controls, KS2 test $\mathrm{p}$ value $<0.05$; controls $\mathrm{n}=8$ animals, 73 cells). The size 
344 and number of blebs varied significantly across the infected RGCs, and bleb size tended to

345 increase with RGC dendritic arbor area (Supplementary Figure 1B, C). A comparison between

346 the P0 and P15 Cpne4 infected RGCs suggest no differences in the bleb areas (P0 mean area=

$3479.95 \pm 1.11 \mathrm{um}^{2}, \mathrm{P} 15$ mean bleb area $\left.=15 \pm 3.75 \mathrm{um}^{2} ; \mathrm{p}=0.2\right)$, suggesting that the process disrupted

348 by Cpne4 overexpression is still active in nearly adult animals (P14), as opposed to the early

349 stages of dendrite formation (P0). A high resolution (Zeiss LSM 880 airy scan) imaging

350 revealed that blebs contained large amounts of Cpne4, as well as cell membranes, as revealed by

351 membrane attached eGFP. Besides the formation of large varicosities or blebs, Cpne4 over-

352 expression into RGCs did not cause any changes in the arbor area or stratification when

353 compared to controls infected with eGFP (Fig.2F, G). Infection of RGCs with AAV1 vectors

354 expressing either C2 domains or vWA domains alone did not cause significant morphological

355 changes or formation of bigger blebs (data not shown).

3.3 Yeast two hybrid analysis reveals Cpne4 vWA domain interaction with Morn2, HCFC1

and Tox3

The domain structure of Copines, and previous work on other family members suggests

361 mediates interactions with other proteins. We therefore used a Gal4 based yeast two hybrid

362 system using the Cpne4 vWA domain as bait protein and an adult mouse retina cDNA library as

363 pray, in order to identify potential retina-specific interactors of Cpne4. Only five clones selected

364 from the initial screening confirmed upon replating (Fig.3A). A colony PCR (Fig.3B) and

365 subsequent Sanger sequencing on the PCR products identified the clones as domains of five

366 proteins- 2 clones of HCFC1, 1 each of Morn2, Tox3 and GAPDH and a mixed clone consisting 
367 of Rhodopsin and GAPDH (Fig.3B). Interestingly, the interacting domain of Tox3 protein 368 contained the HMG box and that for HCFC1 contained an HCF repeat4. For Morn2 the

369 interacting domain consisted of full length of the Morn2 protein and some non-coding sequence

370 on the 5 ' end (Fig.3C). Complete cDNA sequences of the regions of Morn2, HCFC1 and Tox3

371 interacting with Cpne4 are given in the Supplementary document.

\subsection{Cpne4 vWA domain interacts with HCFC1, Morn2 and Mycbp2 in HEK293 cells}

We next sought to validate our candidate Cpne4 interactors (HCFC1, Morn2 and Tox3)

by co-immunoprecipitation. In addition, we surveyed candidate Cpne4-vWA interactors

376 identified in a previous yeast two hybrid analysis using a mouse embryonic cDNA library (26).

377 By exploring our RNA sequencing analysis (1), we found that some of these previously reported

378 candidate Cpne4 interactors (Bicd2, Pitpnm2, Sptbn1 and Mycbp2) were indeed expressed in

379 RGCs (Supp. Fig. 2, Fig. 5A). We therefore cloned the interacting peptide regions from our

380 candidates (HCFC1, Morn2 and Tox3) as well as the matches from the Tomsig et al. study

381 (Bicd2, Pitpnm2, Sptbn1 and Mycbp2) downstream of the Flag tag in an eukaryotic expression

382 vector and co-transfected them with the HA-tagged Cpne4-vWA in HEK293 cells.

383 The colocalization of these proteins was observed by immunostaining (Fig.4B, C, and E;

$384 \mathrm{n}=6,5$ and 4 for Morn2, HCFC1 and Tox3 respectively). While Mycbp2 and Morn2 were

385 localized in the cell body of the cells, Tox3 was always localized in the nuclei (Fig.4E; n=6).

386 Colocalization analysis between Cpne4 and potential interactors showed that both

387 HCFC1 and Morn2 had some degree of colocalization with Cpne4 (mean Spearmann's

388 correlation coefficient $\mathrm{R}=0.19$ for $\mathrm{HCFC} 1, \mathrm{n}=15 \mathrm{ROIs}$; mean $\mathrm{R}=0.32$ for Morn2, $\mathrm{n}=25 \mathrm{ROIs}$ )

389 whereas Tox3 did not (mean $\mathrm{R}=-0.19$, $\mathrm{n}=26$ images; Fig.4F, G). Furthermore, a pull-down 
analysis with Flag antibody showed that the identified Morn2 and HCFC1 domains can interact

392 Of the four Tomsig candidates, only Mycbp2 exhibited a small degree of colocalization with

393 Cpne4 (mean $\mathrm{R}=0.07, \mathrm{n}=13$ images; Fig.4D). Mycbp2 was also pulled down with Cpne4 vWA

394 domain (Fig. 4H, I; n=4). The other candidates - Bicd2, Pitpnm2 and Sptbn1 - were not pulled

395 down with Cpne4 vWA domain (data not shown).

RNA expression as observed by RNA sequencing analysis (Fig.5A) (1) reveals that

HCFC1, Morn2 and Mycbp2 are expressed in RGCs as well as the rest of the retina at E15 and at

P3 (Fig.5A). Immunostaining for HCFC1 (red) in the retina showed that it was localized in the

(Fig.5B; n=2). Immunostaining for Morn2 (green) showed expression in the IPL and GCL in both Brn3b $\mathrm{b}^{\mathrm{WT} / \mathrm{WT}}$ and $\mathrm{Brn} 3 \mathrm{~b}^{\mathrm{KO} / \mathrm{KO}}$ retinas (Fig.5C; $\mathrm{n}=2$ ).

\subsection{Mass Spectrometry analysis of retinal proteins identifies potential Cpne4 interactions.}

Copine4 protein interactions with the vWA domain may be modulated by the $\mathrm{C} 2$

405 domains. In order to identify potential native, full length Copine4 protein binding partners in the

406 retina, we performed pulldown assays of mouse retina protein extracts with GST-vWA or GST-

407 Cpne4 fusion proteins, alongside GST-only controls using Glutathione-Sepharose beads

408 (Fig.6A). Each experimental condition was repeated in triplicate, and Glutathione eluates were

409 loaded onto polyacrylamide gels, separated by electrophoresis, gel bands cut out and processed

410 for LC-MS. A total of 2119 proteins were represented in the LC-MS results by at least one

411 peptide (Supplementary table 1). In order to increase the stringency of our screen, we considered

412 for analysis only proteins that were represented by at least one peptide in all three replicates of at 
413 least one of the pull-down conditions (GST-Cpne4, GST-vWA or GST only; 278 total).

414 Differential expression was performed using the Bioconductor R package DEP (see material and

415 methods), and a fold change of 2 and FDR of $5 \%$ were used as thresholds for differential

416 expression. Based on these criteria, 27 proteins were enriched in both GST-Cpne4 and GST-

417 vWA relative to GST controls, while 180 proteins were significantly enriched in GST-Cpne4 but

418 not GST-vWA samples relative to GST controls (Fig.6A, C, D, Supp. Table 1; n= 3 experiments,

41919 retinas per experiment).

$420 \mathrm{GO}$ analysis performed on the 207 proteins that interacted with Cpne4 or Cpne4-vWA

421 (Supp. Table 2) identified 412 biological processes and 146 molecular functions significantly

422 enriched (FDR $\mathrm{p}<0.05)$. Amongst the top 30 most significantly enriched biological processes

423 (FDR $<=5.21$ e-09) all but two were related to metabolic processes, including glycolysis, ATP

424 or nucleotide metabolism. The two biological processes unrelated to metabolism but interesting

425 from the perspective of Cpne4 involvement in cell morphology and differentiation were

426 "regulation of localization" (FDR $<=2.32$ e-11) and "regulation of transport" (FDR $<=4.29$ e-

427 10). Molecular functions were mostly associated with nucleotide binding, protein binding,

428 enzymatic or catalytic activity. Of the $180 \mathrm{GO}$ cellular components that appeared enriched

429 amongst Cpne4 interactors, at the very top were compartments associated with myelin sheath,

430 cytoplasm, synapse, plasma membrane bounded cell projection, and neuron projection (FDRs $<=$

4312.84 e-19). Interestingly, the highest fold enrichment and low FDR for Cpne4 and its interactors

432 also suggested its localization in the late endosome lumen. A GO pathways analysis (PANTHER

433 GO pathways) reveals that potential Cpne4 interactions participate overwhelmingly in a variety

434 of neuronal seven transmembrane receptor pathways (Muscarinic, Metabotropic Glutamate

435 Receptor, Opioid, Serotonine and Dopamine, etc.) at FDRs $<=8.03$ e-05. PI3 Kinase and 
436 cytoskeletal regulation by Rho GTPase pathways were also significantly enriched. The complete

437 list of all these suggested functions and locations from GO analysis are listed in Supplementary

438 table 2.

439 In order to validate our MS screen, we analyzed several proteins associated with some of

440 the enriched processes: 14-3-3 family of proteins, Map1b, SV2 and Syntaxin-1 (Supp fig. 3).

441 Their association with either GST-vWA or GST-Cpne4 in the retina was confirmed by Western

442 blotting of pulldowns from retinal lysates (Fig.6B). RNA sequencing data showed that the RNA

443 for these candidates was enriched in the retina (Supp. Fig. 2). Using immunohistochemistry, pan

444 14-3-3 and Map1b (red) were found to colocalize in the Cpne4+ RGCs (green; Fig.7A, B; n=3

445 retinas each) in Brn3b WT and KO retina. SV2 and Syntaxin (red) were colocalized in the IPL of

446 both Brn3b WT and KO (Fig. 7C, D; n=3 retinas each).

\section{DISCUSSION}

Amongst Copine family members, Cpne4 has one of the highest mRNA expressions in

451 the retina and is predominantly expressed in the RGC layer (23). Cpne4 over-expression in

452 RGCs lead to formation of large varicosities on dendrites but no other morphological changes to

453 RGC dendritic arbor area or stratification. The morphological defects observed by

454 overexpression in HEK293 cells require a full length Cpne4 protein, as neither C2 domains nor

455 vWA domain can induce extensive process formation in isolation. Cpne4 may be involved in

456 several distinct metabolic and signaling pathways, as revealed by our Yeast two-hybrid and

457 retina proteome interaction studies. Several proteins can interact directly with Cpne4 including

458 Morn2, HCFC1, 14-3-3 family, Map1b, Syntaxin1 and SV2. 


\subsection{Cpne4 regulates cytoskeletal structure in the cells}

It is known that Brn3 transcription factors cause morphological changes in the retina

462 during development and these changes are most likely achieved through their transcriptional

463 targets $(2,23,27,28)$. Cpne4 is one such molecule that is regulated by both Brn3a and Brn3b (1).

464 Results from the current study and previously reported experiments show that over-expression of

465 various Copines in vitro in HEK293 cells leads to elongation of the transfected cells and

466 formation of neurite like structures (23). Transfection with the dominant negative forms of

467 Cpne4 however, did not result in substantial morphology changes. This suggests that the

468 association of C2 domains and vWA domain are critical for inducing phenotypic changes in

469 vitro. Interestingly, the GO analysis of Cpne4 and its interacting proteins suggests its localization

470 in plasma membrane bounded cell projection, and neuron projection, among other locations

471 indicating that it might be involved in cytoskeleton - plasma membrane interactions within the

472 cells.

474 expression, are reminiscent of previously described dendrite swellings, under normal and

475 pathogenetic conditions. In Drosophila larvae, dendrite arborizations of superficial sensory

476 neurons are heavily remodeled during metamorphosis. Prior to pruning, the dendrites tagged for

477 elimination experience marked calcium transients, followed by increased endocytosis and

478 formation of varicosities, like beads on a string. The beaded dendrites are eventually resorbed

479 prior to formation of the new arbor $(29,30)$. Similar large varicosities are also observed on the

480 dendrites of neurons undergoing NMDA-induced excitotoxicity $(31,32)$. 
Other Copines have been previously shown to be involved in morphological changes.

482 Cpne6 mediates cytoskeletal changes in the hippocampus during LTP $(18,19)$. Cpne6 interacts

483 with Rac1 to regulate the Rac1/LimK/Cofilin signaling pathway that further regulates

484 microtubule polymerization and hence affects the spine formation in hippocampal neurons (18).

485 Cpne3 along with Rac1 is required for cell migration during tumor metastasis (33). Copine A, a

486 Copine homolog in Dictyostelium, interacts with actin filaments and regulates chemotaxis and

487 adhesion in these organisms (16). CPNA1, Copine homolog in C. elegans, is located at integrin

488 adhesion sites in the muscle cells and acts as a linker between various myofilament proteins to

489 maintain muscle stability (14).

Taken together, these studies suggest that Copines may mediate membrane - cytoskeletal

491 interactions in a variety of contexts. It is not clear whether the large varicosities on RGCs

492 indicate a role of Cpne4 in dendrite remodeling, or cytoskeletal changes during dendrite

493 development of the retina. Expression of Cpne4 and several other Copine family members

494 increases dramatically during the first two postnatal weeks, a period of intense RGC dendritic

495 arbor growth, differentiation and maturation of dendritic arbor at the time of eye opening.

496 However, the varicosities appeared irrespective of whether the Cpne4 was over-expressed at P0

497 when the eyes are closed and the inner retina is still developing or at P14 when the eyes are open

498 and pruning of the dendrites happens.

$500 \quad 4.2$ Cpne4 and endosomal/lysosomal/autophagosomal pathways

$501 \quad$ Yeast two hybrid analysis on a retina cDNA library indicated Cpne4 interacts with

502 Morn2. Morn2 RNA is expressed in the RGCs and protein expression is seen in the RGCs and

503 dendritic arbor in the IPL. Morn2 consists of a single conserved domain- a MORN (Membrane 
504 Occupation and Recognition Nexus) motif. Morn2 interacts with LC3 to recruit phagosomes

505 against various bacteria in planarian flatworms (34), and is involved in cell membrane

506 engulfment of bacteria and eventually in the invasion of bacteria into the cells (35). Other Morn

507 repeat containing proteins with known functions are junctophilin, retinophilin (also known as

508 Morn4 or Undertaker), PIPK (phosphatidylinositol monophosphate kinase), and Alsin2.

509 We and a previous study (26) have confirmed that Mycbp2 interacts with Cpne4. Mycbp2

510 is a Ubiquitin ligase that contains several conserved domains that are required for a variety of

511 functions (36). Rpm-1, the C. elegans homolog of Mycbp2, regulates AMPA receptor trafficking

512 by regulating the degradation of DLK1 which further regulates Rab5 endosomal protein $(37,38)$.

513 Rab5 endosomes are required for dendritic branching (39).

514 Copine A, in Dictyostelium also associates with vacuoles, endolysosomal organelles and

515 phagosomes in $\mathrm{Ca}^{2+}$ dependent way (40). Cpne6 translocates to clathrin coated vesicles when

$516 \mathrm{Ca}^{2+}$ concentration increases in the cells (6). Previous reports suggest Cpne1 involvement in

517 endosomal and autophagocytosis pathways. Specifically, Cpne1 together with AnnexinA1 and

518 AnnexinA5 and is involved in calcium dependent autophagosomal degradation $(41,42)$. Cpne 1 is

519 also involved in endoproteolysis of NFKB (43).

520 Further studies need to be done to see if Cpne4 is involved in similar function in the

521 retina. One of the top hits for probable sub-cellular location of Cpne4 based on the GO analysis

522 of its interacting proteins is the late endosome lumen. Another possible function of Cpne4 as

523 indicated by GO analysis suggests its role in regulation of transport in the neurons. In this

524 context, the dendritic varicosities induced in RGCs could be the result of disrupted vesicle

525 trafficking. We note that super-resolution imaging of varicosities reveal accumulation of

526 membranes, besides Cpne4 itself. 


\subsection{Cpne4 and neuronal maturation and synapse formation}

Copines were initially discovered in the cell bodies and dendrites of hippocampus

530 neurons and dendrites $(10,17)$ and have been implicated in a variety of functions in the brain.

531 Cpne6 may be involved in long term potentiation $(18,19)$ and suppression of spontaneous

532 neurotransmitter release (44). Cpne1 is upregulated during neural tube closure in mouse

533 embryo, regulates neural stem cell functions during development and it is also required for

534 progenitor cell differentiation of neurons in the hippocampus $(20,21,45)$. The role of Copines in

535 the development or physiology of retina is unknown. But the interactions of Cpne4 with various

536 proteins indicate several possible functions in the retina.

Several protein interactions with Cpne4 were observed with LC-MS analysis. Some of

538 these that were particularly interesting and were expressed in the retina specifically in the inner

539 retina were the 14-3-3 family, Map1b, Syntaxin1 and SV2. 14-3-3 family of proteins is one of

540 the most abundant proteins in the central nervous system. 14-3-3E, that was found to interact

541 with Cpne4, is previously known to bind to doublecortin (Dcx) protein, prevents its degradation

542 which further prevents neurite formation by preventing the microtubules from invading the

543 lamellipodia (46). $14-3-3 \varepsilon$ and $14-3-3 \zeta$ are known to regulate neurogenesis and differentiation of

544 neuronal progenitor cells in the cortex. This is achieved by regulating the catenin/Rho

545 GTPase/Limk1/cofilin signaling pathway to promote F-actin formation (47). 14-3-3y regulates

546 Cpne1 to enhance differentiation of hippocampal progenitor cells and neurite formation in these

547 cells $(21,48)$. Map1b plays an important role in axon guidance and axonal branching during the

548 development of the nervous system $(49,50)$. Synaptic proteins Syntaxin1 and SV2, also

549 interacting with Cpne4, are required in neurotransmitter release at the presynaptic terminal in the 
550 retina $(51,52)$. Interestingly, according to $\mathrm{GO}$ analysis of Cpne4 and its interacting proteins, one

551 of the highest probability of localization of Cpne4 is at the synapse. This also correlates with the

552 previous reports on localization and interaction of Cpne6 with various SNARE proteins on the

553 presynaptic membrane as well as PSD in the postsynaptic membrane $(18,44)$.

554 The yeast two hybrid analysis revealed HCFC1 as an interaction of Cpne4. HCFC1 RNA

555 and protein is also expressed in retina and RGCs. HCFC1 was initially discovered as

556 transcriptional co-activator that along with Oct1 forms a complex with VP16 from Herpes

557 simplex virus and help utilize the transcriptional machinery of the host cell for replicating the

558 virus (53). In neurons, over-expression of HCFC1 leads to neuronal maturation and also limits

559 neurite growth (54). Mutations in HCFC1 protein are associated with Non syndromic Intellectual

560 Disability in humans (55).

561 The functions of Mycbp2 in the central nervous system have been studied extensively

562 before (36). In retina, Mycbp2 RNA expression is observed in RGCs and other cell types and is

563 required for axonal growth cone formation (56). Knocking out Mycbp2 prevents RGC axons

564 from reaching the thalamus and mis-targeted axons in mouse LGN (57-59). Highwire, the

565 homolog of Mycbp2 in Drosophila is also required for synapse formation $(60,61)$.

\section{CONCLUSIONS}

To summarize, Cpne4 over-expression leads to morphological changes in the retina.

570 Cpne4 interacts with multiple proteins and is involved in several different pathways within the

571 cells. This study points to several interesting directions, in relation to possible function of Cpne4 
572 in the retina and the rest of the nervous system. Each specific interaction needs to be explored

573 further to establish their roles in development and functioning of the retina.

574

575 ACKNOWLEDGEMENTS

576 This study was funded by NIH intramural funding support to Tudor C. Badea (EY000504). We

577 want to thank Nadia Parmhans for assistance with genotyping, Jacob Nellissery for sharing the

578 co-immunoprecepitation protocols and Quira Zeidan (NICHD, NIH) for providing the yeast

579 strains AH109 and Y187 and her valuable inputs on growing and mating yeast cells.

\section{REFERENCES}

582 1. Sajgo S, Ghinia MG, Brooks M, Kretschmer F, Chuang K, Hiriyanna S, Wu Z, Popescu

583 O, Badea TC. Molecular codes for cell type specification in Brn3 retinal ganglion cells.

$584 \quad$ Proc Natl Acad Sci U S A. 2017;114(20):E3974-83.

585 2. Muzyka VV, Brooks M, Badea TC. Postnatal developmental dynamics of cell type

586 specification genes in Brn3a/Pou4f1 Retinal Ganglion Cells. Neural Dev. 2018;13(1):1-

$587 \quad 33$

588 3. Liu J, Reggiani JDS, Laboulaye MA, Pandey S, Chen B, Rubenstein JLR, Krishnaswamy

589 A, Sanes JR. Tbr1 instructs laminar patterning of retinal ganglion cell dendrites. Nat

$590 \quad$ Neurosci. 2018 May;21(5):659-70.

591 4. Creutz CE, Tomsig JL, Snyder SL, Gautier MC, Skouri F, Beisson J, Cohen J. The copines, a novel class of C2 domain-containing, calcium-dependent, phospholipid-binding

$5945 . \quad$ Tomsig JL, Creutz CE. Copines: A ubiquitous family of Ca2+-dependent phospholipid- 
binding proteins. Cell Mol Life Sci. 2002;59(9):1467-77.

596 6. Perestenko P, Watanabe M, Beusnard-Bee T, Guna P, McIlhinney J. The second C2domain of copine-2, copine- 6 and copine-7 is responsible for their calcium-dependent membrane association. FEBS J. 2015;282(19):3722-36.

599 7. Whittaker CA, Hynes RO. Distribution and evolution of von Willebrand/integrin A 600 domains: widely dispersed domains with roles in cell adhesion and elsewhere. Mol Biol Cell. 2002 Oct;13(10):3369-87.

602 8. Maitra R, Grigoryev DN, Bera TK, Pastan IH, Lee B. Cloning, molecular characterization, and expression analysis of Copine 8. Biochem Biophys Res Commun. 2003;303(3):842-7.

604 9. Savino M, D’Apolito M, Centra M, Van Beerendonk HM, Cleton-Jansen AM, Whitmore SA, Crawford J, Callen DF, Zelante L, Savoia A. Characterization of copine VII, a new member of the copine family, and its exclusion as a candidate in sporadic breast cancers with loss of heterozygosity at 16q24.3. Genomics. 1999;61(2):219-26.

608 10. Nakayama T, Yaoi T, Yasui M, Kuwajima G. N-copine: a novel two C2-domaincontaining protein with neuronal activity-regulated expression. FEBS Lett. 1998 May;428(1-2):80-4.

611 11. Cowland JB, Carter D, Bjerregaard MD, Johnsen AH, Borregaard N, Lollike K. Tissue expression of copines and isolation of copines I and III from the cytosol of human neutrophils Abstract : Copines are a recently identified group of proteins characterized by two Ca 2 -binding C2- C terminus. Although pEST sequences indicate the. J Leukoc Biol.

616 12. Zhang Y, Chen K, Sloan SA, Bennett ML, Scholze AR, O'Keeffe S, Phatnani HP, 
Maniatis T, Barres BA, Wu JQ. An RNA-sequencing transcriptome and splicing database of glia, neurons, and vascular cells of the cerebral cortex. J Neurosci. 2014 Sep;34(36):11929-47.

621 13. Hua J, Grisafi P, Cheng SH, Fink GR. Plant growth homeostasis is controlled by the arabidopsis BON1 and BAP1 genes. Genes Dev. 2001;15(17):2263-72.

623 14. Warner A, Xiong G, Qadota H, Rogalski T, Vogl AW, Moerman DG, Benian GM. CPNA-1, a copine domain protein, is located at integrin adhesion sites and is required for myofilament stability in Caenorhabditis elegans. Mol Biol Cell. 2013;24(5):601-16.

16. Buccilli MJ, Ilacqua AN, Han M, Banas AA, Wight EM, Mao H, Perry SP, Salter TS, Plays a Role in Chemotaxis and Adhesion. Cells. 2019;8(7):758. $\mathrm{N}$-copine, a member of the two C2 domain protein family, with OS-9, the product of a

635 18. Reinhard JR, Kriz A, Galic M, Angliker N, Rajalu M, Vogt KE, Ruegg MA. The calcium sensor Copine-6 regulates spine structural plasticity and learning and memory. Nat Commun. 2016 May;7:11613.

19. Burk K, Ramachandran B, Ahmed S, Hurtado-Zavala JI, Awasthi A, Benito E, Faram R, 
Apr;28(4):1087-104.

642 20. Greene NDE, Leung K-Y, Wait R, Begum S, Dunn MJ, Copp AJ. Differential protein expression at the stage of neural tube closure in the mouse embryo. J Biol Chem. 2002 Nov;277(44):41645-51.

645 21. Park N, Yoo JC, Ryu J, Hong S-G, Hwang EM, Park J-Y. Copine1 enhances neuronal 646 differentiation of the hippocampal progenitor HiB5 cells. Mol Cells. 2012 Dec;34(6):54954.

22. Liu C-Y, Tsai C-J, Yasugaki S, Nagata N, Morita M, Isotani A, Yanagisawa M, Hayashi Y. Copine-7 is required for REM sleep regulation following cage change or water

23. Goel M, Li T, Badea TC. Differential expression and subcellular localization of Copines in mouse retina. J Comp Neurol. 2019;527(14):2245-62.

653 24. Gan L, Xiang M, Zhou L, Wagner DS, Klein WH, Nathans J. POU domain factor Brn-3b is required for the development of a large set of retinal ganglion cells. Proc Natl Acad Sci U S A. 1996 Apr;93(9):3920-5.

656 25. Zhang X, Smits AH, van Tilburg GB, Ovaa H, Huber W, Vermeulen M. Proteome-wide identification of ubiquitin interactions using UbIA-MS. Nat Protoc. 2018 Mar;13(3):53050.

659 26. Tomsig JL, Snyder SL, Creutz CE. Identification of targets for calcium signaling through 660 the copine family of proteins. Characterization of a coiled-coil copine-binding motif. $\mathrm{J}$ Biol Chem. 2003;278(12):10048-54.

662 27. Badea TC, Cahill H, Ecker J, Hattar S, Nathans J. Distinct Roles of Transcription Factors 663 Brn3a and Brn3b in Controlling the Development, Morphology, and Function of Retinal 
Ganglion Cells. Neuron [Internet]. 2009;61(6):852-64. Available from: http://dx.doi.org/10.1016/j.neuron.2009.01.020

28. Badea TC, Nathans J. Morphologies of mouse retinal ganglion cells expressing transcription factors Brn3a, Brn3b, and Brn3c: analysis of wild type and mutant cells using genetically-directed sparse labeling. Vision Res. 2011 Jan;51(2):269-79.

29. Kanamori T, Yoshino J, Yasunaga KI, Dairyo Y, Emoto K. Local endocytosis triggers dendritic thinning and pruning in Drosophila sensory neurons. Nat Commun [Internet]. 2015;6(May 2014):1-14. Available from: http://dx.doi.org/10.1038/ncomms7515

30. Williams DW, Truman JW. Remodeling dendrites during insect metamorphosis. J Neurobiol. 2005 Jul;64(1):24-33.

674 31. Olney JW, Fuller T, de Gubareff T. Acute dendrotoxic changes in the hippocampus of kainate treated rats. Brain Res. 1979 Oct;176(1):91-100.

676 32. Ahlgren H, Bas-Orth C, Freitag HE, Hellwig A, Ottersen OP, Bading H. The nuclear calcium signaling target, activating transcription factor 3 (ATF3), protects against dendrotoxicity and facilitates the recovery of synaptic transmission after an excitotoxic promotes tumor metastasis by enhancing cell migration via copine-III and Rac1. Oncogene. 2018;37(40):5416-34. 
planarians identifies MORN2 as a key component in LC3-associated phagocytosis and resistance to bacterial infection. Cell Host Microbe [Internet]. 2014;16(3):338-50. Available from: http://dx.doi.org/10.1016/j.chom.2014.08.002

35. McGuire AM, Cochrane K, Griggs AD, Haas BJ, Abeel T, Zeng Q, Nice JB, Macdonald set of Fusobacterium species. MBio. 2014;5(6):1-11.

693 36. Po MD, Hwang C, Zhen M. PHRs: bridging axon guidance, outgrowth and synapse development. Curr Opin Neurobiol. 2010;20(1):100-7.

37. Grill B, Bienvenut W V., Brown HM, Ackley BD, Quadroni M, Jin Y. C. elegans RPM-1

Rab GTPase GLO-1. Neuron. 2007;55(4):587-601.

38. Park EC, Glodowski DR, Rongo C. The ubiquitin ligase RPM-1 and the p38 MAPK PMK-3 regulate AMPA receptor trafficking. PLoS One. 2009;4(1).

39. Satoh D, Sato D, Tsuyama T, Saito M, Ohkura H, Rolls MM, Ishikawa F, Uemura T. Rab5-endosomes. Nat Cell Biol. 2008 Oct;10(10):1164-71.

40. Damer CK, Bayeva M, Hahn ES, Rivera J, Socec CI. Copine A, a calcium-dependent membrane-binding protein, transiently localizes to the plasma membrane and intracellular 
710 42. Ghislat G, Knecht E. New Ca2+-dependent regulators of autophagosome maturation. Commun Integr Biol. 2012;5(4):308-11.

712 43. Ramsey CS, Yeung F, Stoddard PB, Li D, Creutz CE, Mayo MW. Copine-I represses NF$713 \quad \kappa B$ transcription by endoproteolysis of p65. Oncogene. 2008;27(25):3516-26.

714 44. Liu P, Khvotchev M, Li YC, Chanaday NL, Kavalali ET. Copine-6 Binds to SNAREs and

45. Kim TH, Sung S-E, Cheal Yoo J, Park J-Y, Yi G-S, Heo JY, Lee J-R, Kim N-S, Lee DY. Res Commun. 2018 Jan;495(1):168-73.

46. Cornell B, Wachi T, Zhukarev V, Toyo-Oka K. Regulation of neuronal morphogenesis by Genet. 2016 Oct;25(20):4405-18.

47. Toyo-oka K, Wachi T, Hunt RF, Baraban SC, Taya S, Ramshaw H, Kaibuchi K, Schwarz 81. in HiB5 hippocampal progenitor cells. Exp Cell Res. 2017 Jul;356(1):85-92. 
Dec;151(6):1169-78.

734 50. Bouquet C, Soares S, von Boxberg Y, Ravaille-Veron M, Propst F, Nothias F.

Microtubule-associated protein 1B controls directionality of growth cone migration and axonal branching in regeneration of adult dorsal root ganglia neurons. J Neurosci. 2004 Aug;24(32):7204-13.

51. Sherry DM, Mitchell R, Standifer KM, du Plessis B. Distribution of plasma membraneassociated syntaxins 1 through 4 indicates distinct trafficking functions in the synaptic

52. Wang MM, Janz R, Belizaire R, Frishman LJ, Sherry DM. Differential distribution and developmental expression of synaptic vesicle protein 2 isoforms in the mouse retina. $\mathbf{J}$ Comp Neurol. 2003 May;460(1):106-22.

53. Wysocka J, Herr W. The herpes simplex virus VP16-induced complex: The makings of a

54. Huang L, Jolly LA, Willis-Owen S, Gardner A, Kumar R, Douglas E, Shoubridge C, 
http://dx.doi.org/10.1016/j.ajhg.2013.07.022

757 56. Lewcock JW, Genoud N, Lettieri K, Pfaff SL. The Ubiquitin Ligase Phr1 Regulates Axon

Outgrowth through Modulation of Microtubule Dynamics. Neuron. 2007;56(4):604-20.

759 57. Bloom AJ, Miller BR, Sanes JR, DiAntonio A. The requirement for Phr1 in CNS axon

760 tract formation reveals the corticostriatal boundary as a choice point for cortical axons.

$761 \quad$ Genes Dev. 2007;21(20):2593-606.

762 58. Culican SM, Bloom AJ, Weiner JA, DiAntonio A. Phr1 regulates retinogeniculate targeting independent of activity and ephrin-A signalling. Mol Cell Neurosci [Internet].

59. Vo BQ, Bloom AJ, Culican SM. Phr1 is required for proper retinocollicular targeting of nasal-dorsal retinal ganglion cells. Vis Neurosci. 2011;28(2):175-81.

767 60. Wan HI, DiAntonio A, Fetter RD, Bergstrom K, Strauss R, Goodman CS. Highwire regulates synaptic growth in Drosophila. Neuron. 2000;26(2):313-29.

769 61. Nakata K, Abrams B, Grill B, Goncharov A, Huang X, Chisholm AD, Jin Y. Regulation of a DLK-1 and p38 MAP kinase pathway by the ubiquitin ligase RPM-1 is required for presynaptic development. Cell. 2005;120(3):407-20.

772 62. Chuang K, Nguyen E, Sergeev Y, Badea TC Novel Heterotypic Rox Sites for Combinatorial Dre Recombination Strategies. G3 (Bethesda). 2015 Dec 29;6(3):559-71. 


\section{Figure1: Cpne4 and Cpne4 dominant negative transfections in HEK293: (A) Map of the}

780 Flex construct. (B) Map of domain structure of Cpne4 shows three domains of the Cpne4

781 protein- two C2 domains (blue) and a vWA domain (purple). The location of the three Cpne4

782 plasmid constructs are shown by full length Cpne4 construct (orange), vWA domain construct

783 (brown) and C2 domains construct (green). The binding of the two Cpne4 antibodies- N-terminal

784 and C-terminal antibodies on the Cpne4 protein are indicated by yellow triangles. (C)

785 Representative images of HEK293 cells transfected with expression constructs for full length

786 Cpne4 (top row), C2 domains construct (middle row) and vWA domain construct (bottom row).

787 The cells were counterstained for eGFP (green), HA (red) and N-terminal or C-terminal Cpne4

788 (white) antibodies. Scale bar: 50um.

\section{Figure2: Cpne4 virus infections lead to 'bleb' formation on dendrites of Brn3b+ RGCs}

791 (A, B) Intraocular injections of AAV1-Cpne4 viruses were done in P0 or P14 Brn3b ${ }^{\text {Cre/WT }}$ mice

792 eyes. Blebs were seen occasionally on dendrites of Cpne4 infected RGCs. For controls, AAV1-

793 eGFP was similarly injected in P0 Brn3b $b^{\text {Cre/WT }}$ mice $(C, D)$. No blebs were observed in the

794 control infected RGCs. Airy scan images for one of the blebs (A1) and adjoining dendrites (A2).

795 (E) Measurement of the 'bleb' areas for Cpne4 infected RGCs as compared to regular

796 varicosities on controls shows a significant difference between them while no difference between

797 P0 and P15 Cpne4 infected retinas. (F, G) Areas and lamination measurements indicate no

798 differences between the Cpne4 transfected RGCs as compared to control transfected RGCs. (ID:

799 inner distance- distance between outermost tip of RGC dendrites and the INL; IPL: width of the

800 IPL). Scale bar for A-C: 50um; Scale bar for A', A”: 5 um. 
803 A Gal4 based yeast two hybrid analysis on the adult mouse retina cDNA library with Cpne4

804 vWA domain as bait protein showed five positive interactions (blue colonies). (B) Gel image of

805 colony PCR performed on the the positive clones from the yeast two hybrid shows the

806 corresponding interacting DNA sequences. The identity of these DNA sequences as confirmed

807 by the Sanger sequencing followed by BLAST analysis are shown as labels on the lanes. (C)

808 Sequences of some of the interacting peptides as identified by Sanger sequencing followed by

809 BLAST analysis are shown- HCFC1 (green); Mycbp2 (orange) and Morn2 (purple). The relative

810 positions of the actual interacting region on the respective protein is indicated by arrows and any

811 protein domains in that region and surrounding regions are also labeled.

813 Figure4: Cpne4 Adomain was pulled down with HCFC1, Morn2 and Mycbp2, but not by

814 Tox3: (A) Map of a Flag clone consisting of a Flag tag followed by the interacting regions of

815 HCFC1, Morn2, Mycbp2 and Tox3. (B-E) Representative images of HEK293 cells co-infected

816 with the expression construct for Cpne4vWAdomain (HA-Cpne4vWA) and Flag- tagged

817 interacting protein. (F, G) Box plots and violin plots indicate the Spearmann's correlation

818 coefficient for cololcalization between Morn2, HCFC1, Tox3 or Mycbp2 with Cpne4. (H)

819 Western blot images of pull down from co-transfected HEK293 cells using Flag antibody show

820 the total lysate $(I)$ and the co-immunoprecipitated $(C)$ proteins. Cpne4-vWA (green; $31 \mathrm{kDa})$

821 pulled down Flag- HCFC1 (23.5kDa), Morn2 (21kDa), Mycbp2 (42kDa) and not Tox3 (66kDa).

822 Scale bar: 50um. 
824 Figure5: Expression of Morn2, HCFC1 and Mycbp2 in retina: (A) RNA sequencing data for

825 Morn2, Mycbp2 and HCFC1 in different brain areas, Brn3a WT and KO RGCs, Brn3b WT and

826 KO RGCs and rest of the retina. (B) HCFC1 (red) immunostaining in retina indicates some

827 expression in RGCs in both the WT and Brn3b KO retinas. There is some co-labeling with

828 Cpne4 (green) in the RGC cell bodies (arrows). (C) Morn2 (green) immunolabeling in the retina

829 indicated mostly dendritic labeling in the IPL in both Brn3b WT and KO retinas. There is also

830 some Morn2 labeling in the OPL of WT that is reduced in the Brn3b KO. Scale bar: 50um.

Figure6: Pulldown with GST-Cpne4 or GST-CpneAdomain and LC-MS: (A) Representative SDS-PAGE gel for retina pull downs using GST- Cpne4, GST- Cpne4- vWA and GST only

$834(n=3)$. (B) Representative Western blot image of some of the interactors from the pull down 835 experiment- pan 14-3-3 (30kDa); Map1b (325kDa); SV2 (95kDa) and Syntaxin1 (33kDa) as

836 compared to GST (28kDa) control. (C) Volcano plot for proteins that had a significant

837 interaction with GST- Cpne4 as compared to GST control. (D) Volcano plot for proteins that had 838 a significant interaction with GST-Cpne4- vWA as compared to GST control. 


\begin{tabular}{|c|c|c|c|}
\hline Antigen & immunogen & Antibody details & $\begin{array}{r}\text { Dilution used } \\
\text { for IHC (and WB) }\end{array}$ \\
\hline $\begin{array}{l}\text { C- terminal } \\
\text { Cpne4 }\end{array}$ & EVYESSRTLA & $\begin{array}{l}\text { made in house, } \\
\text { rabbit polyclonal }\end{array}$ & $\begin{array}{c}1: 2000 \\
(1: 1000 \text { for } \mathrm{WB})\end{array}$ \\
\hline Brn3a & $\begin{array}{l}\text { Human Brn3a aa186-224 } \\
\text { protein } 10 \text { fusion (pGEMEX) }\end{array}$ & $\begin{array}{l}\text { Millipore, mouse } \\
\text { monoclonal, Cat\# } \\
\text { MAB1585 }\end{array}$ & $1: 20$ \\
\hline Brn3b & $\begin{array}{c}\text { Human Brn3b aa184-252 GST } \\
\text { fusion }\end{array}$ & $\begin{array}{l}\text { made in house, } \\
\text { rabbit polyclonal }\end{array}$ & $1: 20$ \\
\hline mouse HA & CYPYDVPDYASL & $\begin{array}{l}\text { Covance, mouse } \\
\text { monoclonal, clone } \\
\text { 16B12 }\end{array}$ & 1:100 \\
\hline rabbit HA & YPYDVPDYASL & $\begin{array}{c}\text { Cell Signaling } \\
\text { Technologies, rabbit } \\
\text { polyclonal, Cat\# } 3724\end{array}$ & $1: 100$ \\
\hline GFP & Recombinant full length GFP & $\begin{array}{l}\text { Abcam, chicken } \\
\text { polyclonal, Cat\# } \\
\text { ab13970 }\end{array}$ & $1: 700$ \\
\hline
\end{tabular}




\begin{tabular}{|c|c|c|c|}
\hline Morn2 & $\begin{array}{c}\text { Recombinant protein against the } \\
\text { epitope- } \\
\text { MNGFGRLEHFSGAVYEGQFK } \\
\text { DNMFHGLGTYTFPNGAKYT } \\
\text { G } \\
\text { NFNENRVEGEGEYTDIQGLE } \\
\text { WS } \\
\text { GNFHFTAAPDLKLK }\end{array}$ & $\begin{array}{l}\text { Sigma prestige } \\
\text { antibodies, rabbit } \\
\text { polyclonal, Cat\# } \\
\text { HPA057815 }\end{array}$ & $1: 100$ \\
\hline HCF1 & $\begin{array}{l}\text { aa 206-251 mapping near the N- } \\
\text { terminus of human } \mathrm{HCF} 1\end{array}$ & $\begin{array}{c}\text { Santa Cruz } \\
\text { Biotechnology, mouse } \\
\text { monoclonal, } \\
\text { Cat\# sc-390950 }\end{array}$ & $1: 50$ \\
\hline Map1b & $\begin{array}{l}\text { aa 6-31 at the N-terminus of human } \\
\text { MAP-1B }\end{array}$ & $\begin{array}{c}\text { Santa Cruz } \\
\text { Biotechnology, mouse } \\
\text { monoclonal, } \\
\text { Cat\# sc-365668 }\end{array}$ & $\begin{array}{r}1: 50 \\
(1: 250)\end{array}$ \\
\hline Pan 14-3-3 & $\begin{array}{l}\text { aa } 1-30 \text { at the } \mathrm{N} \text {-terminus of } \\
\text { human pan } 14-3-3\end{array}$ & $\begin{array}{c}\text { Santa Cruz } \\
\text { Biotechnology, mouse } \\
\text { monoclonal, Cat\# sc- } \\
1657\end{array}$ & $\begin{array}{r}1: 50 \\
(1: 500)\end{array}$ \\
\hline
\end{tabular}




\begin{tabular}{|c|c|c|c|}
\hline SV2 & Purified synaptic vesicles & $\begin{array}{l}\text { Developmental } \\
\text { Studies Hybridoma } \\
\text { Bank, DSHB, of the } \\
\text { University of Iowa, } \\
\text { USA, mouse } \\
\text { monoclonal }\end{array}$ & $\begin{array}{r}1: 500 \\
(1: 2000)\end{array}$ \\
\hline Syntaxin 1 & $\begin{array}{l}\text { Recombinant protein corresponding } \\
\text { to AA } 1 \text { to } 262 \text { from rat Syntaxin1A } \\
\text { (UniProt } \\
\text { Id: P32851) }\end{array}$ & $\begin{array}{l}\text { Synaptic systems, } \\
\text { mouse monoclonal, } \\
\text { Cat\# } 110011\end{array}$ & $\begin{array}{r}1: 200 \\
(1: 2500)\end{array}$ \\
\hline GST & $\begin{array}{c}26 \mathrm{kDa} \text { GST specific domain of a } \\
\text { fusion protein encoded by a } \\
\text { pGEX.3X recombinant vector }\end{array}$ & $\begin{array}{c}\text { Santa Cruz } \\
\text { Biotechnology, rabbit } \\
\text { polyclonal, } \\
\text { Cat\# sc-459 }\end{array}$ & $(1: 750)$ \\
\hline
\end{tabular}




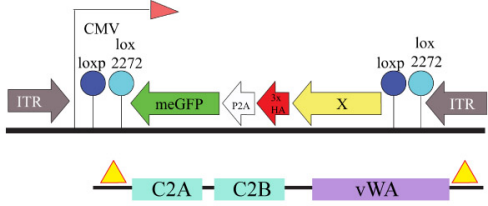

B

Constructs

Cpne4 vWA

C2

C
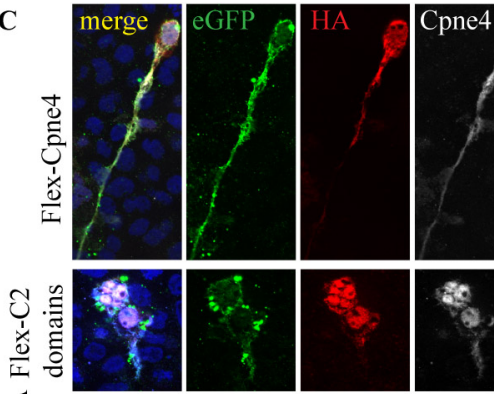

273 532 325

$\sum_{\frac{1}{3}}^{\frac{\pi}{\pi}}$

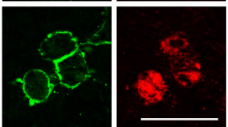

557 


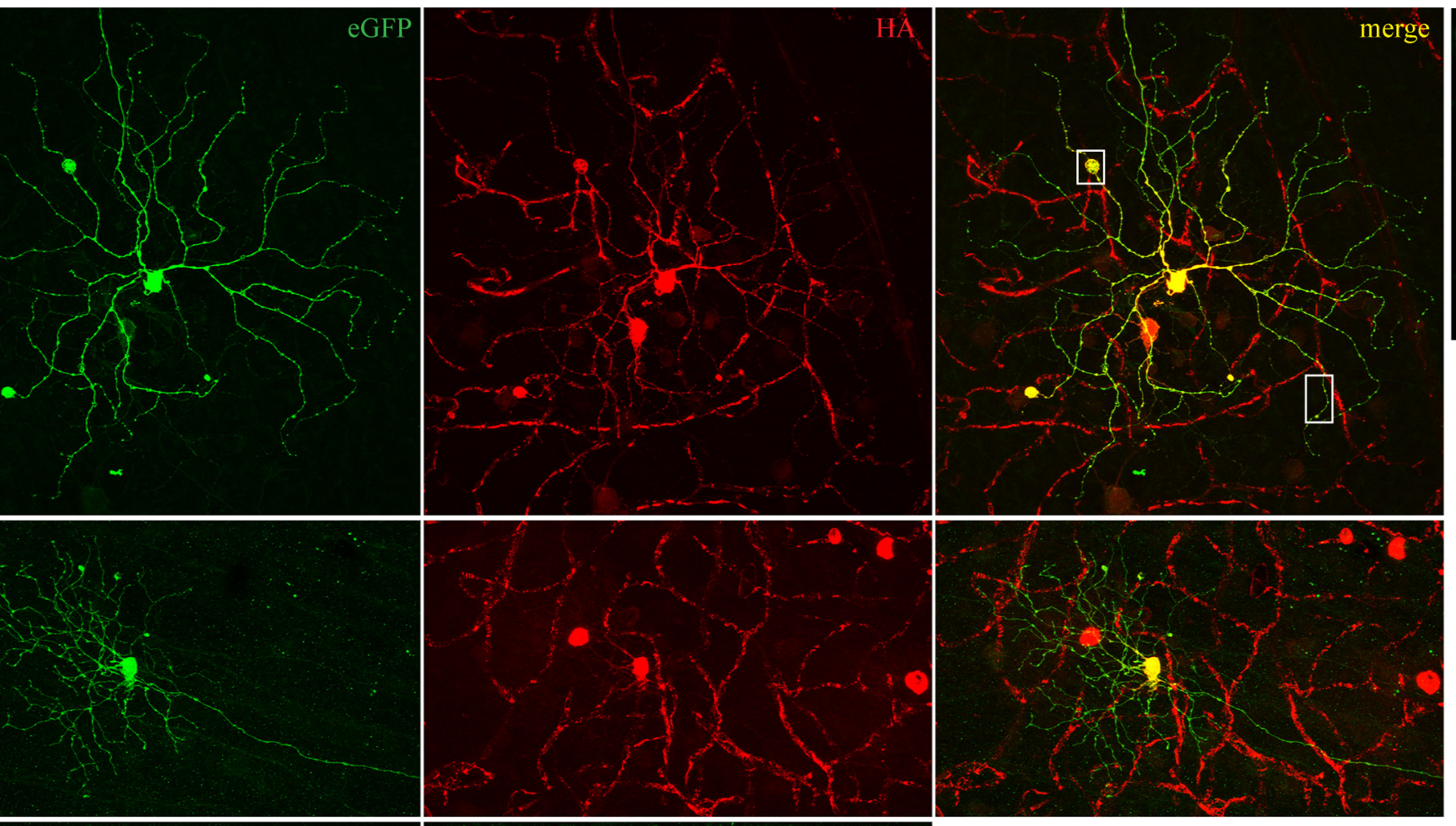

C

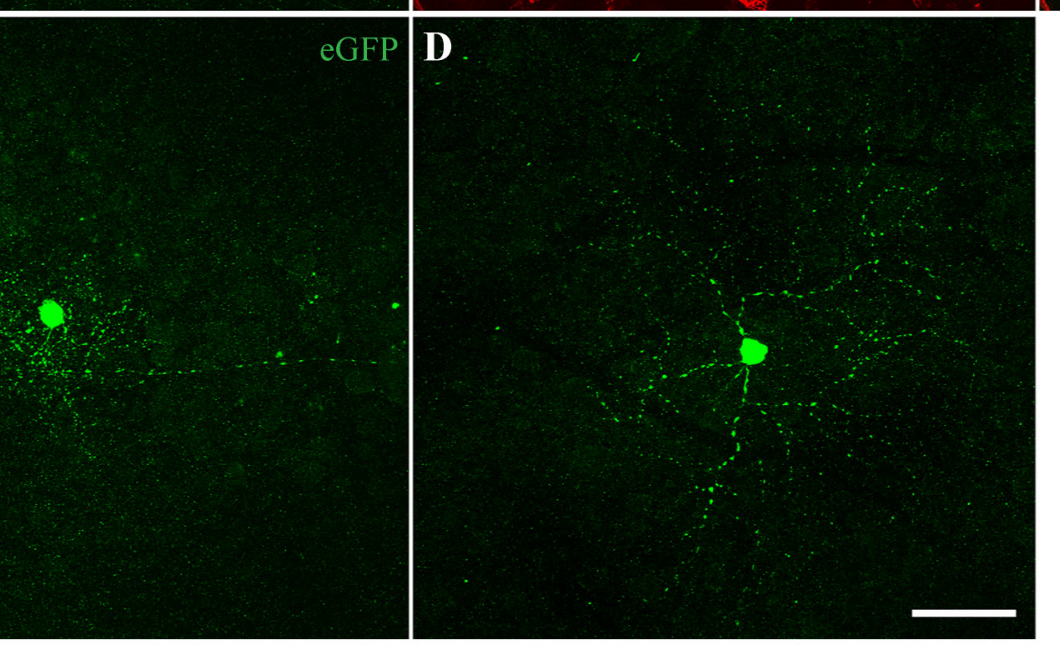

F 100000

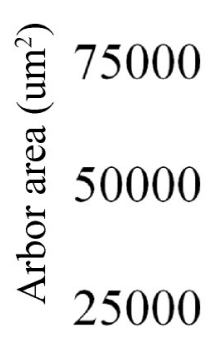

0
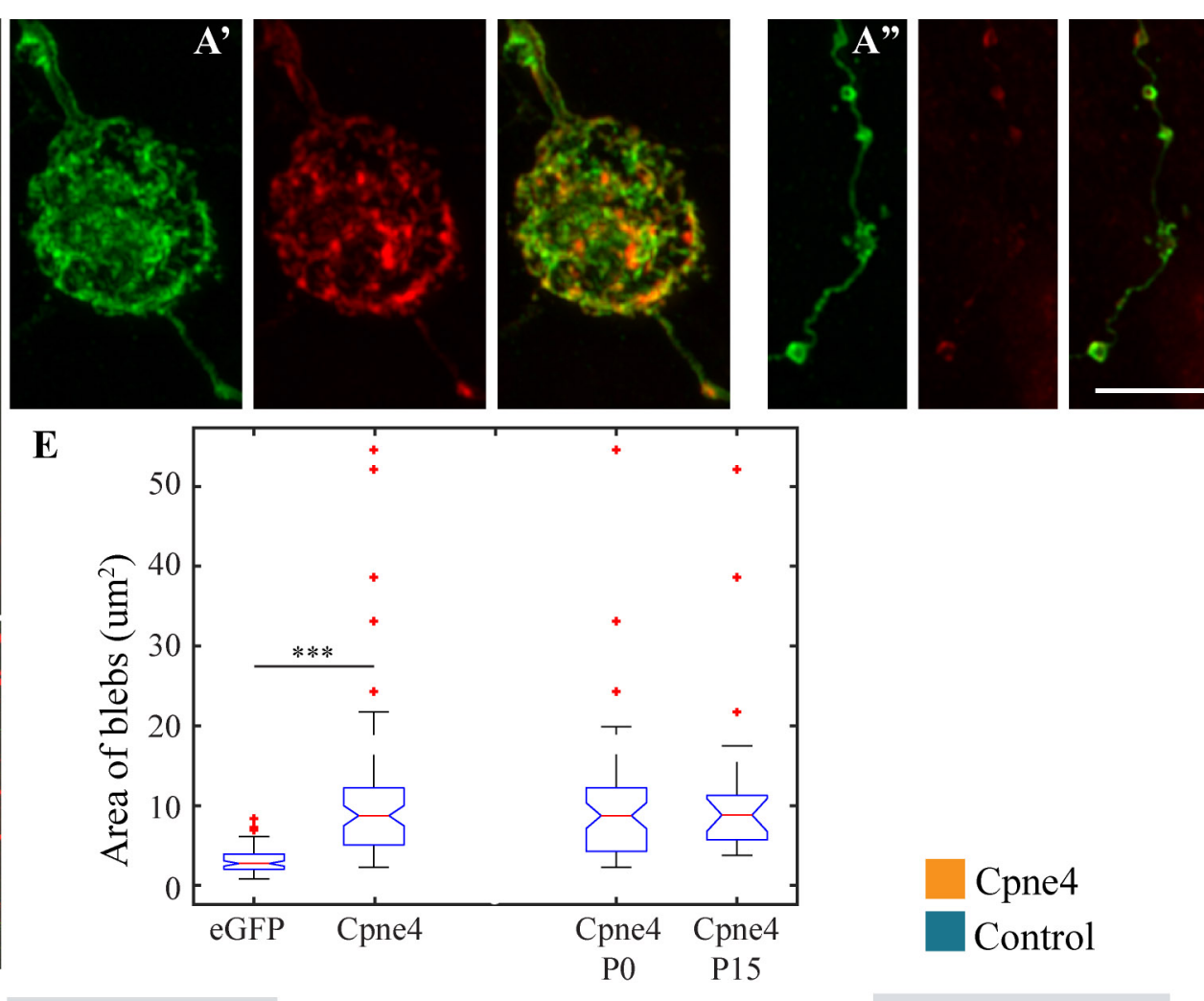

Cpne4 $\stackrel{\leftrightarrows}{\Theta}^{0.75}$ 商 0.5 0.25 0

$$
\begin{array}{llcll}
0 & 0.25 & 0.5 & 0.75 & 1 \\
\mathrm{ID} / \mathrm{IPL} & &
\end{array}
$$


Morn2 HCFC1 GAPDH Tox3 GAPDH arana positive negative control control

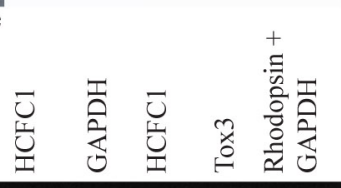

B

$500 \mathrm{bp}$ 400 300 200 100

Tox3

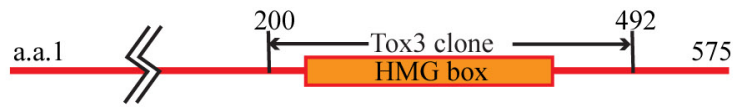

Morn2

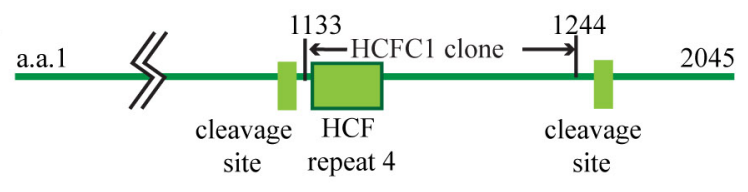

$\mathrm{HCFCl}$

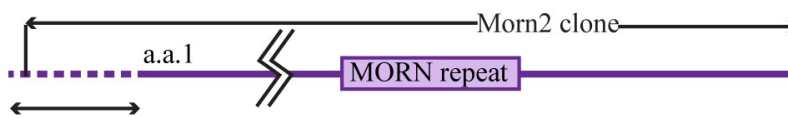

non-coding

sequence on mRNA 
A $\quad$ IP: $\quad$ IP:

B

IP: GST- GST-

GST vWA Cpne4

$150 \mathrm{kDa}-$ 100 -

$75^{-}$

$50-$

$37-$

25

-GST-Cpne4

-GST-vWA

$20-$

-GST

input 123

$$
\begin{aligned}
-\quad- & \text { pan 14-3-3 } \\
-\quad- & \text { Map1b } \\
-\quad & \text { SV2 } \\
+\quad & \text { SyntaxinI } \\
& \text { GST-Cpne4 } \\
\text { GST-Cpne4-vWA } & \text { GST }
\end{aligned}
$$

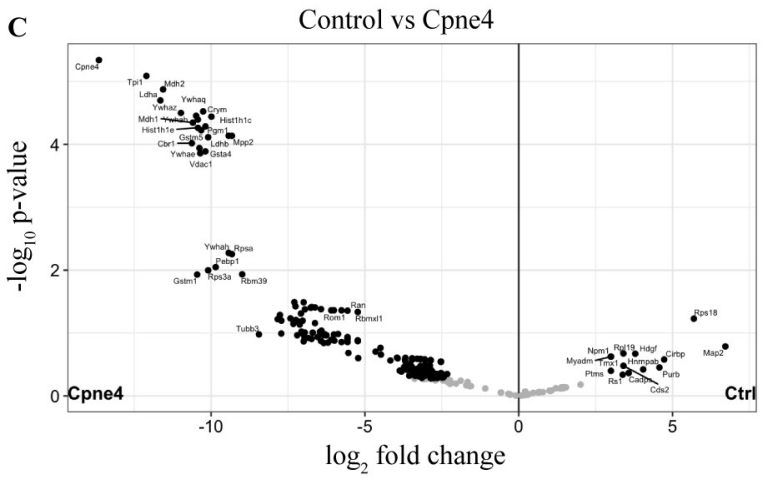

D Control vs vWA

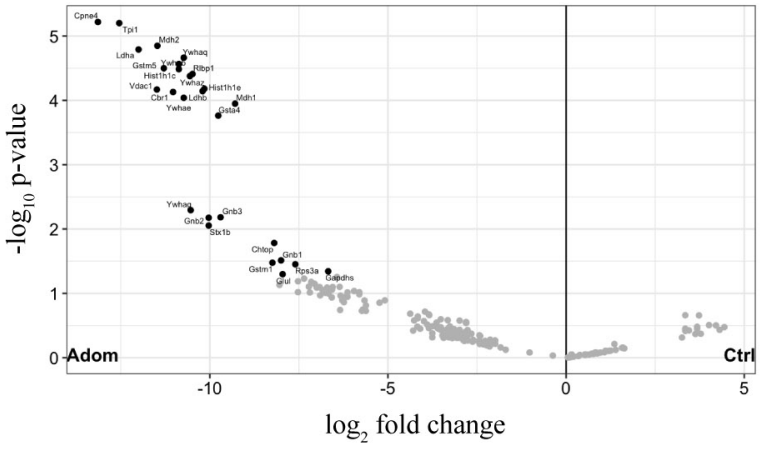




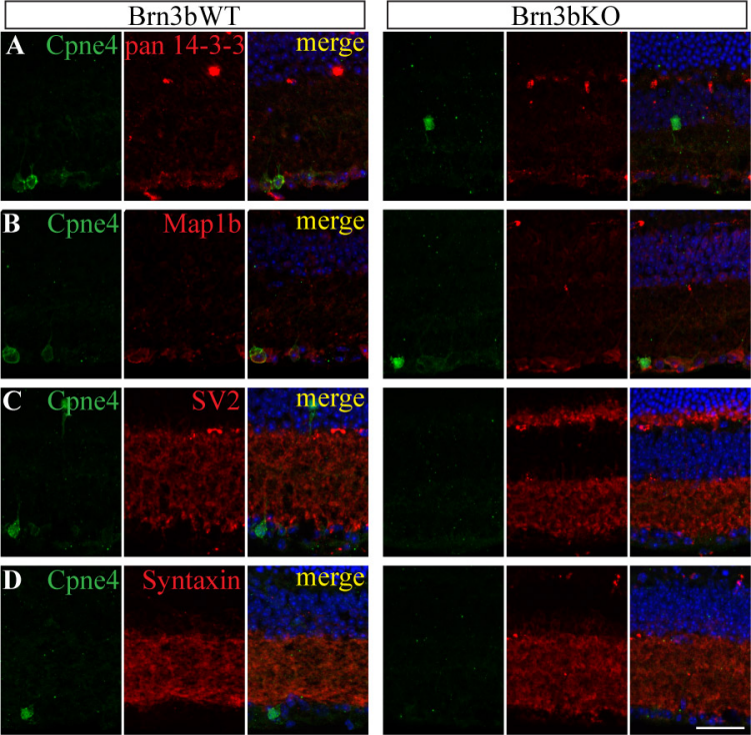




\section{SUPPLEMENTARY RESULTS}

cDNA sequence of the interactors from the yeast two hybrid interactions-

Morn2:

AAGCAGTGGTATCAACGCAGAGTGGCCATTATGGCCGGGGGAGGCCCGCTCTGTCC TAGAGCCCAGCTCCCTCCAGCCTGCCGAGCTGGCGAGCCTAGGGGAATCGCAGAGT CGCGAAAGCCTGTCCTTCACGTCTCCATCAACTTCGTCAGAAGTATATAAGATCAAA TTTATATTTCCAAATGGAGACACATACGATGGCGACTGCACAAGAACTACCTCTGGG ATCTGTGAGAGAAACGGGACAGGCACGCACACCACGCCGAACGGGATTGTCTACAC AGGAAGCTGGAAAGATGACAAGATGAATGGCTTTGGAAGACTTGAACATTTTTCGG GCGCTGTGTATGAAGGACAGTTTAAGGACAACATGTTTCATGGACTGGGGACTTACA CATTCCCAACTGGGGCAAAGTACACTGGAAATTTCAATGAAAATAGGGTAGAAGGT GAGGGAGAATACACTGACACCCAAGGCCTGCAGTGGTGTGGTAACTTCCACTTCAC AGCTGCCCCCGGCCTGAAACTAAAGCTCTACATGTAGACCTGCTGCCTTAACGCTGA GATGTGGCCTCTGCAACCCCCCTTAGGCAAAGCAACTGAACCTTCTGCTAAAGTGAC CTGCCCTCTTCCGTAAGTCCAATAAAGTTGTCATGCACCCACACCTTTTTGAATTATG TATTGTATGTGTGTGAGCTCATGTGTGTTTATGTGCATGTGTGTGCACATGTGCTTGT ATGCATGTGTGTGTGTGTGTCTGTGTGTGAGTACTTGTTCATGCAGGTACATGTGGA AGGCAGAAGTTGATAATAAAATGCCTTTTTCAGGCTAGAACATGCTTTTAATCTCAG CACTCAGGAGAGGCAGGCAGATCTCCTGAGTTGCAGGACAGCCATGGCTACACATC TTGAAAAAGAAAAAAAAAATCCTTTTCAATCCCTTTTTACTTTTTTTTTTTTTTAAAG ACATGGTCTTACTATATGGCCATGGTTGGCCTAAAACTCACTGTGTAGACCAGGTTG TCCTCAAACTCACAAAGATCTACCTGTTTCTTCTTACCAAGTGCTAGGATTAAAGGT GTTTGGTCACTAGATTCAATCTTTTCACCTCATTTGTTCCTCAGTGAACCTGGAGCTC ACTGTTTCTGCTAATTTGGCTGGCCAGTGGCCCCCAGGAAACGCCCATCTCTGCTCA CCCCCAGCTGAGCACGGAGGTTAGGCACGTACCAGCGCGCCTGGAGAGGGAATCAG GGTCTTCACATTTGCAGGGAAAGCACTTTGCCCACTAAGGCATTTCCCCGACTCTTG AATTGTATCTTCATTAGGAAATGACTTTAAATAAATTGTGTGAATCAATTCAGAGTT TATGTATGTTTTAAAATTGGAGCAGTACGGTCCGAATGGTGGCTAAGTCACTATCTA ACTCTGCACCTCAACTTTTGTCAGCCCCCATGTCGGCCGCCTCGGCCTCTAGA 
HCFC1:

AAGCAGTGGTATCAACGCAGAGTGGCCATTATGGCCGGGGCGAGACACTCGTCGTA CCACTAACACCCCCACTGTAGTGCGGATCACTGTGGCTCCTGGGGCATTGGAGAGA GTCCAGGGTACCGTGAAGCCTCAGTGCCAAACCCAGCAGACCAACATGACCACCAC CACCATGACTGTGCAGGCCACTGGAGCTCCATGCTCAGCTGGCCCCCTGCTTAGGCC AAGTGTGGCACTGGAGTCTGGGAGCCACAGCCCTGCCTTTGTGCAACTAGCCCTTCC AAGTGTCAGAGTTGGGCTAAGTGGCCCCAGCAGCAAGGACATGCCCACAGGGCGCC AACCAGAGACATATCATACTTACACAACTAATACCCCCATGTCGGCCGCCTCGGCCT CTAGAATCCCGGCGCATGTCGGCCGCCTCGGCCTCTAGA

Tox3:

AAGCAGTGGTATCAACGCAGAGTGGCCATTATGGCCGGGACCTCCAGCAAGCAAAT CAGCCACTCCCTCTCCTTCCAGCTCTATCAACGAAGAGGATGCTGATGATGCAAACA GAGCCATTGGAGAGAAAAGAACAGCCCCAGATTCTGGCAAGAAGCCCAAGACTCCA AAGAAAAAGAAAAAGAAAGATCCCAACGAGCCTCAGAAGCCAGTGTCAGCATATG CCCTGTTTTTCAGAGATACACAGGCTGCAATTAAGGGTCAAAACCCCAACGCAACCT TCGGAGAAGTCTCGAAAATCGTAGCATCTATGTGGGACAGCCTTGGGGAGGAGCAA AAGCAGGTATATAAAAGGAAAACAGAAGCTGCCAAGAAAGAATACTTGAAGGCCC TGGCTGCCTACCGGGCTAGCCTCGTTTCCAAGGCTGCTGCTGAATCTGCAGAAGCCC AGACCATCCGTTCTGTCCAGCAGACTCTGGCATCAACCAACCTGACATCCTCCCTCC TCCTGAACACATCACTGTCTCAACATGGGACAGTCCCAGCCTCACCTCAGACTCTTC CACAGTCACTCCCTAGGTCAATTGCCCCCAAACCCTTAACCATGAGACTACCCATGA GCCAGATCGTCACATCAGTCACCATTGCAGCCAACATGCCCTCGAACATTGGGGCTC CACTGATAAGTTCCATGGGGACGACCATGGTTGGTTCAGCAACCTCCACCCAGGTGA GCCCTTCGGTGCAAACCCAGCAGCATCAGATGCAGTTGCAGCAGCAACAGCAGCAG CAGCAGCAGATGCAGCAGATGCAGCAGCAGCAGTTACAGCAGCACCAAATGCATCA GCAGATCCAGCAGCAGATGCAGCAGCAGCATTTTCAGCATCACATGCAGCAGCACC TGCAGCAGCAGCATGTCGGCCGCCTCGGCCTCTAGA 


\section{SUPPLEMENTARY FIGURE LEGENDS}

Supplementary Figure1: Varicosity area measurements: (A) Box plots showing the areas of the regular varicosities on eGFP infected controls, regular size varicosities on Cpne4 infected RGCs, all varicosities (regular and large blebs) on Cpne4 infected RGCs and all varicosities on P0 and P15 Cpne4 infected RGCs. (B) Plot showing correlation between arbor area and varicosity area for Cpne4 infected RGCs. (C) Plot showing correlation between arbor area and varicosity area for eGFP control infected RGCs.

Supplementary Figure2: RNA sequencing data: RNA sequencing data showing Map1b, Stx1b; 14-3-3 family- Ywhab, Ywhaz, Ywhae, Ywhag, Ywhaq; SV2a; Bicd2; Pitpnm2 and Sptbn1 in different brain areas, Brn3a WT and KO RGCs, Brn3b WT and KO RGCs, rest of the retina and visual brain areas.

Supplementary Figure3: Graphs for interesting MS proteins: (A) Bar graphs showing log2 fold change as found in MS analysis for 3 different conditions- Cpne4 vs Adom (Cpne4-vWA), Ctrl (control) vs Adom and Ctrl vs Cpne4 for the proteins Cpne4, 14-3-3 family- Ywhaz, Ywhab, Ywhae, Ywhaq and Ywhag. (B) Bar graphs showing log2 fold change as found in MS analysis for 3 different conditions- Cpne4 vs Adom, Ctrl vs Adom and Ctrl vs Cpne4 for the proteins Cpne4, SV2a, Map1b and Stx1b (Syntaxin 1b).

\section{SUPPLEMENTARY TABLE TITLES}

Supplementary Table1: Complete LC-MS data including the list of enriched proteins (Cpne4 vs control, Adomain vs control or Cpne4 vs Adomain; A) and raw data (B) containing the PSM (peptide spectrum match) values for each protein for each replicate ( $\mathrm{n}=3$ experiments)

Supplementary Table2: GO analysis of enriched proteins from LC-MS data listing most probable biological processes (A), molecular function (B), cellular component (C) and significant pathways from PANTHER analysis (D). 
